\title{
Synchronization between Different Networks with Time-Varying Delay and Its Application in Bilayer Coupled Public Traffic Network
}

\author{
Wenju Du, ${ }^{1}$ Jiangang Zhang, ${ }^{2}$ Yinzhen $\mathrm{Li}^{1}{ }^{1}$ and Shuang Qin ${ }^{2}$ \\ ${ }^{1}$ School of Traffic and Transportation, Lanzhou Jiaotong University, Lanzhou 730070, China \\ ${ }^{2}$ School of Mathematics and Physics, Lanzhou Jiaotong University, Lanzhou 730070, China \\ Correspondence should be addressed to Wenju Du; duwenjuok@126.com
}

Received 19 January 2016; Revised 9 April 2016; Accepted 24 April 2016

Academic Editor: Ricardo Aguilar-López

Copyright (C) 2016 Wenju Du et al. This is an open access article distributed under the Creative Commons Attribution License, which permits unrestricted use, distribution, and reproduction in any medium, provided the original work is properly cited.

\begin{abstract}
In order to study the dynamic characteristics of urban public traffic network, this paper establishes the conventional bus traffic network and the urban rail traffic network based on the space $R$ modeling method. Then regarding these two networks as the subnetwork, the paper presents a new bilayer coupled public traffic network through the transfer relationship between subway and bus, and this model well reflects the connection between the passengers and bus operating vehicles. Based on the synchronization theory of coupling network with time-varying delay and taking "Lorenz system" as the network node, the paper studies the synchronization of bilayer coupled public traffic network. Finally, numerical results are given to show the impact of public traffic dispatching, delayed departure, the number of public bus stops between bus lines, and the number of transfer stations between two traffic modes on the bilayer coupled public traffic network balance through Matlab simulation.
\end{abstract}

\section{Introduction}

Synchronization of complex networks is an important subject of study dynamics of complex networks; in recent years, many scholars have studied in depth the synchronization of complex networks [1-10]. However, most studies are only focused on the single networks synchronization and not many on the study of synchronization between two different networks. Li et al. [11] focus on two unidirectionally coupled networks and derive analytically a criterion for the synchronization of these two networks. Tang et al. [12] designed effective adaptive controllers and addressed the theoretical analysis of synchronization between two complex networks with nonidentical topological structures. Chen et al. [13] presented a general network model for two complex networks with time-varying delay coupling and derived a synchronization criterion by using the adaptive controllers. Wang et al. [14] designed an adaptive controller to achieve synchronization between two different complex networks with time-varying delay coupling. Sun et al. [15] investigated the linear generalized synchronization between two complex networks. In [16] the outer synchronization between two complex networks with discontinuous coupling is studied and the sufficient conditions for complete outer synchronization and generalized outer synchronization are obtained. Sun and Li [17] investigated the generalized outer synchronization between two uncertain dynamical networks with a novel feature that the couplings of each network are unknown functions. Based on Lyapunov stability theory and Barbalat's lemma, they obtained two sufficient criteria for generalized outer synchronization with or without time delay. And two types of synchronization between two coupled networks with interactions, including inner synchronization inside each network and outer synchronization between two networks with the adaptive controllers, are investigated in [18]. They designed the adaptive controllers to realize the inner and outer synchronization simultaneously and obtained a theorem for the outer synchronization with the adaptive controllers. However, they did not consider timevarying delay in their work, but the existence of delay is 
inevitable in real life and delay can affect many synchronous phenomena, so the study of synchronization between two different complex networks with time-varying delay coupling is particularly important.

As one of the important research tools, complex networks have been widely used in urban traffic system [19-22]. Urban public transport network is an actual typical complex network, and there has been a lot of research and analysis of public transport network. However, most studies just investigated the static characteristics of complex network and its stability, such as the topology properties study of traffic network, the study of reliability and robustness of the network, and the research of structure optimization. And there are few literatures that studied the dynamic characteristics of urban public traffic network. Because the urban public traffic network own its unique characteristics, to analyze its dynamic characteristics is very necessary.

With the development of economy and society, the city size and population are gradually increasing, and the resident trips are also increasing. Limited city traffic space difficultly meets the increasing traffic demand and the traffic congestion is becoming more and more serious. Just through control and optimization of conventional public traffic to solve this problem has no significant effect, and the emergence of urban rail traffic has greatly made up for many shortcomings of conventional public traffic. The conventional public traffic and urban rail traffic both belong to urban public transportation system, and each has its unique superiority. The conventional public traffic is of lower operating cost and wide coverage and flexible. The urban rail traffic has the characteristics of higher speed, large freight volume, being fast, safety, and causing less environmental pollution. And it also has its own shortcomings. Therefore, strengthening the effective cooperation and transfer join between conventional public traffic and urban rail traffic can help to improve the operating efficiency of the whole city public transportation system and maximum to meet the needs of passengers. However, at present most studies only focused on a single conventional public traffic network or single urban rail traffic network.

This paper presents a new complex network synchronization model and designs an adaptive controller to make the two different networks achieve synchronization based on the LaSalle invariable principle. In addition, we construct a new bilayer coupled public traffic network model by using the space $R$ modeling method. And the synchronization problem of this model is studied by using the synchronization theory of two different complex networks with time-varying delay coupling. At last, we discussed the impact of the artificial scheduling, the number of the public stations between two traffic lines, the number of the transfer stations between conventional bus lines and subway lines, and delayed departure to the new bilayer coupled public traffic network model's synchronous ability.

The paper is organized as follows. The synchronization theory of two different complex networks with time-varying delay coupling is presented in Section 2. In Section 3, a new bilayer public traffic coupled network model is established. In Section 4, the synchronization problem of the bilayer coupled public traffic network model is investigated. Simulation results are given to show the impact of the artificial scheduling, the number of the public stations between two traffic lines, the number of the transfer stations between conventional bus lines and subway lines, and delayed departure to the bilayer coupled public traffic network balance in Section 5. In Section 6, we conclude the paper.

\section{Synchronization between Two Different Complex Networks with Time-Varying Delay Coupling}

Consider two networks with time-varying delay coupling, and the fact that they both consist of same nodes can be described by

$$
\begin{aligned}
\dot{x}_{i}(t)= & f\left(x_{i}(t)\right)+\varepsilon_{1} \sum_{j=1}^{N_{1}} a_{i j} \Gamma_{1} x_{j}(t-\tau(t)) \\
& +\mu \sum_{j=1}^{N_{2}} c_{i j} y_{j}(t-\tau(t)), \quad i=1,2, \ldots, N_{1}, \\
\dot{y}_{i}(t)= & g\left(y_{i}(t)\right)+\varepsilon_{2} \sum_{j=1}^{N_{2}} b_{i j} \Gamma_{2} y_{j}(t-\tau(t)) \\
& +\mu \sum_{j=1}^{N_{1}} d_{i j} x_{j}(t-\tau(t))+u_{i}, \quad i=1,2, \ldots, N_{2},
\end{aligned}
$$

where $x_{i}=\left(x_{i 1}, x_{i 2}, \ldots, x_{i n}\right)^{T} \in R^{n}, y_{i}=\left(y_{i 1}, y_{i 2}, \ldots, y_{i n}\right)^{T} \in$ $R^{n}$ are the state vectors of the $i$ th node in network (1) and network (2), $\dot{x}_{i}(t), \dot{y}_{i}(t)$ are the dynamic equation for a single node, $f(\cdot), g(\cdot): R^{n} \rightarrow R^{n}$ are the nonlinear continuous differentiable vector function, $N_{1}, N_{2}$ are the number of nodes of networks (1) and (2), $\Gamma_{1}, \Gamma_{2} \in R^{n \times n}$ is a constant matrix linking the coupled variables, the constant $\varepsilon_{1}, \varepsilon_{2}$ denotes the inner coupling strengths of network (1) and network (2), respectively, the constant $\mu$ denotes the outer coupling strength, and $\tau(t)$ is the time-varying coupling delay. Coupling matrices $A=\left(a_{i j}\right) \in R^{N_{1} \times N_{1}}, B=\left(b_{i j}\right) \epsilon$ $R^{N_{2} \times N_{2}}$ are, respectively, the inner connection matrices of the driving network (1) and the response network (2), where $a_{i i}=-\sum_{j=1, i \neq j}^{N_{1}} a_{i j}, b_{i i}=-\sum_{j=1, i \neq j}^{N_{2}} b_{i j}$, and $a_{i j}\left(b_{i j}\right)$ are defined as follows: if there is a connection from node $j$ to node $i(i \neq j)$, then $a_{i j}\left(b_{i j}\right)>0(i \neq j)$; otherwise $a_{i j}\left(b_{i j}\right)=0(i \neq j)$. The matrices $C=\left(c_{i j}\right) \in R^{N_{1} \times N_{2}}, D=\left(d_{i j}\right) \in R^{N_{2} \times N_{1}}$ are the coupling matrix between two networks, where $c_{i j}, d_{i j}$ are defined as follows: if there is a connection from node $i$ (belongs to network (1)) to node $j$ (belongs to network (2)), then $c_{i j}>0$; otherwise $c_{i j}=0$; if there is a connection from node $i$ (belongs to network (2)) to node $j$ (belongs to network (1)), then $d_{i j}>0$; otherwise $d_{i j}=0$, and $u_{i}(t)$ is the controller for node $i$ to be designed according to the specific network structures $A$ and $B$. Without loss of generality, assume that 
$N_{1}>N_{2}$; that is, networks (1) and (2) have the different number of nodes.

Definition 1. Let $x_{i}\left(t, X_{0}\right)\left(i=1,2, \ldots, N_{1}\right)$ and $y_{i}\left(t, Y_{0}\right.$, $\left.u_{i}\right)\left(i=1,2, \ldots, N_{2}\right)$ be the solutions of the networks (1) and (2), where $X_{0}=\left(x_{1}^{0}, x_{2}^{0}, \ldots, x_{N_{1}}^{0}\right)^{T} \in R^{n N_{1}}, Y_{0}=$ $\left(y_{1}^{0}, y_{2}^{0}, \ldots, y_{N_{2}}^{0}\right)^{T} \in R^{n N_{2}}$, and $f, g: \Omega \rightarrow R^{n}$ are the continuously differentiable mappings with $\Omega \subseteq R^{n}$. If there is a nonempty open subset $\Lambda \subseteq \Omega$, with $x_{i}^{0}, y_{i}^{0} \in \Lambda$, so when $t \geq 0$ such that $x_{i}\left(t, X_{0}\right)\left(1 \leq i \leq N_{1}\right), y_{i}\left(t, Y_{0}, u_{i}\right)(1 \leq i \leq$ $\left.N_{2}\right) \in \Omega$, and

$$
\lim _{t \rightarrow \infty}\left\|y_{i}\left(t, Y_{0}, u_{i}\right)-x_{i}\left(t, X_{0}\right)\right\|=0, \quad\left(i=1,2, \ldots, N_{2}\right)
$$

then complex networks (1) and (2) are said to realize synchronization.

Assumption 2. For function $f(x)$ there exists a positive constant $L$ such that

$$
\|f(y(t))-f(x(t))\|=L\|y(t)-x(t)\|
$$

where, $\forall x(t), y(t) \in R^{n}$.

Assumption 3. Here $\tau(t)$ is a differential function with $0 \leq$ $\dot{\tau}(t) \leq \xi<1$. Obviously, this assumption includes constant time delay as a special case.

Lemma 4. For arbitrary $x, y \in R^{n}, \eta>0,2 x^{T} y \leq \eta x^{T} x+$ $(1 / \eta) y^{T} y$ is established.

Theorem 5. Suppose that Assumptions 2 and 3 hold. One selects the following controllers:

$$
\begin{aligned}
u_{i}= & f\left(y_{i}(t)\right)-g\left(y_{i}(t)\right)+\varepsilon_{1} \sum_{j=1}^{N_{2}} a_{i j} \Gamma_{1} y_{j}(t-\tau(t)) \\
& -\varepsilon_{2} \sum_{j=1}^{N_{1}} b_{i j} \Gamma_{2} x_{j}(t-\tau(t))+\mu \sum_{j=1}^{N_{1}} c_{i j} x_{j}(t-\tau(t)) \\
& -\mu \sum_{j=1}^{N_{2}} d_{i j} y_{j}(t-\tau(t))+\varepsilon_{1} \sum_{j=N_{2}+1}^{N_{1}} a_{i j} \Gamma_{1} x_{j}(t-\tau(t)) \\
& +\varepsilon_{2} \sum_{j=N_{2}+1}^{N_{1}} b_{i j} \Gamma_{2} x_{j}(t-\tau(t)) \\
& -\mu \sum_{j=N_{2}+1}^{N_{1}}\left(c_{i j}+d_{i j}\right) x_{j}(t-\tau(t))-g_{i} e_{i}(t)
\end{aligned}
$$

and then driving network (1) and response network (2) can realize synchronization under controllers (5), where $\dot{g}_{i}=$ $k_{i}\left\|e_{i}\right\|^{2}$ and $k_{i}$ is a positive constant, $i=1,2, \ldots, N_{2}$.
Proof. Define the errors vector by $e_{i}(t)=y_{i}(t)-x_{i}(t), i=$ $1,2, \ldots, N_{2}$; then the error system can be described by

$$
\begin{aligned}
& \dot{e}_{i}(t)=g\left(y_{i}(t)\right)-f\left(x_{i}(t)\right)+\varepsilon_{2} \sum_{j=1}^{N_{2}} b_{i j} \Gamma_{2} y_{j}(t-\tau(t)) \\
& -\varepsilon_{1} \sum_{j=1}^{N_{1}} a_{i j} \Gamma_{1} x_{j}(t-\tau(t))+\mu \sum_{j=1}^{N_{1}} d_{i j} x_{j}(t-\tau(t)) \\
& -\mu \sum_{j=1}^{N_{2}} c_{i j} y_{j}(t-\tau(t))+f\left(y_{i}(t)\right)-g\left(y_{i}(t)\right) \\
& +\varepsilon_{1} \sum_{j=1}^{N_{2}} a_{i j} \Gamma_{1} y_{j}(t-\tau(t)) \\
& -\varepsilon_{2} \sum_{j=1}^{N_{1}} b_{i j} \Gamma_{2} x_{j}(t-\tau(t))+\mu \sum_{j=1}^{N_{1}} c_{i j} x_{j}(t-\tau(t)) \\
& -\mu \sum_{j=1}^{N_{2}} d_{i j} y_{j}(t-\tau(t)) \\
& +\varepsilon_{1} \sum_{j=N_{2}+1}^{N_{1}} a_{i j} \Gamma_{1} x_{j}(t-\tau(t)) \\
& +\varepsilon_{2} \sum_{j=N_{2}+1}^{N_{1}} b_{i j} \Gamma_{2} x_{j}(t-\tau(t)) \\
& -\mu \sum_{j=N_{2}+1}^{N_{1}}\left(c_{i j}+d_{i j}\right) x_{j}(t-\tau(t))-g_{i} e_{i}(t) \\
& =f\left(y_{i}(t)\right)-f\left(x_{i}(t)\right)+\varepsilon_{1} \sum_{j=1}^{N_{2}} a_{i j} \Gamma_{1} e_{j}(t-\tau(t)) \\
& +\varepsilon_{2} \sum_{j=1}^{N_{2}} b_{i j} \Gamma_{2} e_{j}(t-\tau(t)) \\
& -\sum_{j=1}^{N_{2}} \mu\left(c_{i j}+d_{i j}\right) e_{j}(t-\tau(t))-g_{i} e_{i}(t) .
\end{aligned}
$$

Choose the Lyapunov-Krasovskii candidate as follows:

$$
\begin{aligned}
V(t)= & \frac{1}{2} \sum_{i=1}^{N_{2}} e_{i}^{T}(t) e_{i}(t) \\
& +\frac{1}{2(1-\xi)} \sum_{i=1}^{N_{2}} \int_{t-\tau(t)}^{t} e_{i}^{T}(\alpha) e_{i}(\alpha) d \alpha \\
& +\frac{1}{2} \sum_{i=1}^{N_{2}} \frac{1}{k_{i}}\left(g_{i}-\bar{g}\right)^{2},
\end{aligned}
$$


where $\bar{g}$ is a sufficiently larger positive constant which is to be determined. Using Assumptions 2 and 3 and Lemma 4, we can get the following formula by derivation of (7):

$$
\begin{aligned}
& \dot{V}(t)=\sum_{i=1}^{N_{2}} e_{i}^{T}(t) \dot{e}_{i}(t)+\frac{1}{2(1-\xi)} \sum_{i=1}^{N_{2}} e_{i}^{T}(t) e_{i}(t) \\
& -\frac{1-\dot{\tau}(t)}{2(1-\xi)} \sum_{i=1}^{N_{2}} e_{i}^{T}(t-\tau(t)) e_{i}(t-\tau(t))+\sum_{i=1}^{N_{2}} \frac{1}{k_{i}}\left(g_{i}\right. \\
& -\bar{g}) \dot{g}_{i}=\sum_{i=1}^{N_{2}} e_{i}^{T}(t)\left[f\left(y_{i}(t)\right)-f\left(x_{i}(t)\right)\right. \\
& +\varepsilon_{1} \sum_{j=1}^{N_{2}} a_{i j} \Gamma_{1} e_{j}(t-\tau(t))+\varepsilon_{2} \sum_{j=1}^{N_{2}} b_{i j} \Gamma_{2} e_{j}(t-\tau(t)) \\
& \left.-\sum_{j=1}^{N_{2}} \mu\left(c_{i j}+d_{i j}\right) e_{j}(t-\tau(t))-g_{i} e_{i}(t)\right]+\frac{1}{2(1-\xi)} \\
& \cdot \sum_{i=1}^{N_{2}} e_{i}^{T}(t) e_{i}(t)-\frac{1-\dot{\tau}(t)}{2(1-\xi)} \sum_{i=1}^{N_{2}} e_{i}^{T}(t-\tau(t)) e_{i}(t \\
& -\tau(t))+\sum_{i=1}^{N_{2}} \frac{1}{k_{i}}\left(g_{i}-\bar{g}\right) \dot{g}_{i} \leq \sum_{i=1}^{N_{2}}(L-\bar{g})\left\|e_{i}(t)\right\|^{2} \\
& +\varepsilon_{1} \sum_{i=1}^{N_{2}} \sum_{j=1}^{N_{2}} e_{i}^{T}(t) a_{i j} \Gamma_{1} e_{j}(t-\tau(t)) \\
& +\varepsilon_{2} \sum_{i=1}^{N_{2}} \sum_{j=1}^{N_{2}} e_{i}^{T}(t) b_{i j} \Gamma_{2} e_{j}(t-\tau(t)) \\
& -\mu \sum_{i=1}^{N_{2}} \sum_{j=1}^{N_{2}} e_{i}^{T}(t)\left(c_{i j}+d_{i j}\right) e_{j}(t-\tau(t))+\frac{1}{2(1-\xi)} \\
& \cdot \sum_{i=1}^{N_{2}} e_{i}^{T}(t) e_{i}(t)-\frac{1-\dot{\tau}(t)}{2(1-\xi)} \sum_{i=1}^{N_{2}} e_{i}^{T}(t-\tau(t)) e_{i}(t \\
& -\tau(t))=\sum_{i=1}^{N_{2}}(L-\bar{g})\left\|e_{i}(t)\right\|^{2}+\varepsilon_{1} \sum_{j=1}^{n} e_{j}^{T}(t) \\
& \cdot \gamma_{1 j} A^{\prime} e_{j}(t-\tau(t))+\varepsilon_{2} \sum_{j=1}^{n} e_{j}^{T}(t) \gamma_{2 j} B e_{j}(t-\tau(t)) \\
& -\mu \sum_{j=1}^{n} e_{j}^{T}(t)\left(C^{\prime}+D^{\prime}\right) e_{j}(t-\tau(t))+\frac{1}{2(1-\xi)} \\
& \cdot \sum_{i=1}^{N_{2}} e_{i}^{T}(t) e_{i}(t)-\frac{1-\dot{\tau}(t)}{2(1-\xi)} \sum_{j=1}^{n} e_{j}^{T}(t-\tau(t)) e_{j}(t \\
& -\tau(t)) \leq \sum_{i=1}^{N_{2}}(L-\bar{g})\left\|e_{i}(t)\right\|^{2}+\sum_{j=1}^{n} \frac{\gamma_{1 j}^{2}}{2} e_{j}^{T}(t)\left(\varepsilon_{1} A^{\prime}\right)
\end{aligned}
$$

$$
\begin{aligned}
& \cdot\left(\varepsilon_{1} A^{\prime}\right)^{T} e_{j}(t)+\sum_{j=1}^{n} \frac{\gamma_{2 j}^{2}}{2} e_{j}^{T}(t)\left(\varepsilon_{2} B\right)\left(\varepsilon_{2} B\right)^{T} e_{j}(t) \\
& -\sum_{j=1}^{n} \frac{1}{2} e_{j}^{T}(t)\left(\mu C^{\prime}+\mu D^{\prime}\right)\left(\mu C^{\prime}+\mu D^{\prime}\right)^{T} e_{j}(t) \\
& +\frac{1}{2(1-\xi)} \sum_{i=1}^{N_{2}} e_{i}^{T}(t) e_{i}(t)+\left(\frac{1}{2}-\frac{1-\dot{\tau}(t)}{2(1-\xi)}\right) \sum_{j=1}^{n} e_{j}^{T}(t \\
& -\tau(t)) e_{j}(t-\tau(t)) \leq \sum_{i=1}^{N_{2}}(L-\bar{g})\left\|e_{i}(t)\right\|^{2} \\
& +\sum_{j=1}^{n} \frac{\gamma_{1 j}^{2}}{2} e_{j}^{T}(t)\left(\varepsilon_{1} A^{\prime}\right)\left(\varepsilon_{1} A^{\prime}\right)^{T} e_{j}(t)+\sum_{j=1}^{n} \frac{\gamma_{2 j}^{2}}{2} e_{j}^{T}(t) \\
& \cdot\left(\varepsilon_{2} B\right)\left(\varepsilon_{2} B\right)^{T} e_{j}(t)-\sum_{j=1}^{n} \frac{1}{2} e_{j}^{T}(t)\left(\mu C^{\prime}+\mu D^{\prime}\right)\left(\mu C^{\prime}\right. \\
& \left.+\mu D^{\prime}\right)^{T} e_{j}(t)+\frac{1}{2(1-\xi)} \sum_{i=1}^{N_{2}} e_{i}^{T}(t) e_{i}(t) \leq \sum_{i=1}^{N_{2}}(L-\bar{g} \\
& \left.+\frac{1}{2(1-\xi)}\right)\left\|e_{i}(t)\right\|^{2}+\gamma_{j} \sum_{j=1}^{n} e_{j}^{T}(t) P e_{j}(t) \leq e^{T}(t) \\
& \cdot\left\{\left[L-\bar{g}+\frac{1}{2(1-\xi)}+\max _{1 \leq j \leq n}\left(\gamma_{j}\right) \lambda_{\max }(P)\right] I_{n N_{2}}\right\} \\
& \cdot e(t)=\mathbf{e}^{T}(t) Q \mathbf{e}(t),
\end{aligned}
$$

where $\mathbf{e}(t)=\left(e_{1}(t), e_{2}(t), \ldots, e_{N_{2}}(t)\right)^{T} \in R^{n N_{2}}$ and $\gamma_{1 j}, \gamma_{2 j}$ are the $j$ th diagonal elements of $\boldsymbol{\Gamma}_{1}, \boldsymbol{\Gamma}_{2}$, respectively. Consider

$$
\begin{aligned}
\gamma_{j}= & \max \left\{\frac{\gamma_{1 j}^{2}}{2}, \frac{\gamma_{2 j}^{2}}{2}, \frac{1}{2}\right\}, \\
P= & \left(\varepsilon_{1} A^{\prime}\right)\left(\varepsilon_{1} A^{\prime}\right)^{T}+\left(\varepsilon_{2} B\right)\left(\varepsilon_{2} B\right)^{T} \\
& +\left(\mu C^{\prime}+\mu D^{\prime}\right)\left(\mu C^{\prime}+\mu D^{\prime}\right)^{T}, \\
Q= & {\left[L-\bar{g}+\frac{1}{2(1-\xi)}+\max _{1 \leq j \leq n}\left(\gamma_{j}\right) \lambda_{\max }(P)\right] I_{n N_{2}}, }
\end{aligned}
$$

where $\lambda_{\max }(P)$ is the largest eigenvalue of matrix $P, I_{n N_{2}}$ is the unit $n \times N_{2}$ matrix, $A^{\prime}, C^{\prime}, D^{\prime}$ are the $N_{2}$ order principal minor determinant of matrices $A, C, D$, respectively. Obviously, there exists a sufficiently large positive constant $\bar{g}$ such that the symmetry matrix $Q$ is negative definite; namely, $\dot{V}(t)<0$. Here, the largest invariant set contained in set $E=\{\dot{V}(t)=$ $0\}=\left\{\mathbf{e}(t)=0, i=1,2, \ldots, N_{2}\right\}$ can be described as

$$
M=\left\{(\mathbf{e}, \mathbf{g}) \in R^{n N_{2}} \times R^{N_{2}}: \mathbf{e}=0, \dot{\mathbf{g}}=0\right\},
$$


where $\mathbf{g}=\left(g_{1}, g_{2}, \ldots, g_{N_{2}}\right)^{T}$. According to the LaSalle invariance principle, starting with arbitrary initial values, the trajectory asymptotically converges to the largest invariant $M$ which implies that $\lim _{t \rightarrow \infty} e_{i}(t)=0, i=1,2, \ldots, N_{2}$, so driving network (1) and response network (2) realized synchronization. The proof is completed.

Corollary 6. If networks (1) and (2) have the same node number, driving network (1) and response network (2) can realize synchronization by using the following controllers:

$$
\begin{aligned}
u_{i}= & f\left(y_{i}(t)\right)-g\left(y_{i}(t)\right)+\varepsilon_{1} \sum_{j=1}^{N} a_{i j} \Gamma_{1} y_{j}(t-\tau(t)) \\
& -\varepsilon_{2} \sum_{j=1}^{N} b_{i j} \Gamma_{2} x_{j}(t-\tau(t))+\mu \sum_{j=1}^{N} c_{i j} x_{j}(t-\tau(t)) \\
& -\mu \sum_{j=1}^{N} d_{i j} y_{j}(t-\tau(t))-g_{i} e_{i}(t),
\end{aligned}
$$

where $e_{i}(t)=y_{i}(t)-x_{i}(t), \dot{g}_{i}=k_{i}\left\|e_{i}\right\|^{2}$, and $k_{i}$ is a positive constant, $i=1,2, \ldots, N$.

Corollary 7. If networks (1) and (2) have the same node dynamic, then using the controllers

$$
\begin{aligned}
u_{i}= & \varepsilon_{1} \sum_{j=1}^{N_{2}} a_{i j} \Gamma_{1} y_{j}(t-\tau(t))-\varepsilon_{2} \sum_{j=1}^{N_{1}} b_{i j} \Gamma_{2} x_{j}(t-\tau(t)) \\
& +\mu \sum_{j=1}^{N_{1}} c_{i j} x_{j}(t-\tau(t))-\mu \sum_{j=1}^{N_{2}} d_{i j} y_{j}(t-\tau(t)) \\
& +\varepsilon_{1} \sum_{j=N_{2}+1}^{N_{1}} a_{i j} \Gamma_{1} x_{j}(t-\tau(t)) \\
& +\varepsilon_{2} \sum_{j=N_{2}+1}^{N_{1}} b_{i j} \Gamma_{2} x_{j}(t-\tau(t)) \\
& -\mu \sum_{j=N_{2}+1}^{N_{1}}\left(c_{i j}+d_{i j}\right) x_{j}(t-\tau(t))-g_{i} e_{i}(t),
\end{aligned}
$$

can make driving network (1) and response network (2) realize synchronization, where $e_{i}(t)=y_{i}(t)-x_{i}(t), \dot{g}_{i}=k_{i}\left\|e_{i}\right\|^{2}$, and $k_{i}$ is a positive constant, $i=1,2, \ldots, N_{2}$.

\section{A New Bilayer Public Traffic Coupled Network Model}

Urban public traffic network can be regarded as a complex network which consists of different stops and lines. There are mainly three kinds of modeling methods to establish the urban public traffic network: space $L$ modeling method, space $P$ modeling method, and space $R$ modeling method
[23, 24]. Using space $L$ modeling method to establish public traffic stations networks, taking the bus station as the nodes of network, the two bus stations have edge if they are adjacent in a bus line. Using space $P$ modeling method to establish public traffic transfer networks, also taking the bus station as the nodes of network, the two bus stations have edge if there are direct bus lines between them. Using space $R$ modeling method to establish public traffic roads networks, taking bus lines as the network's nodes, the two bus lines have edge if there exist same bus stops between them. This paper established a new bilayer coupled public traffic network model based on the existing modeling methods and the modeling idea as follows:

(1) Taking conventional public traffic lines and urban rail traffic lines as the network's nodes, then based on the space $R$ modeling method and established conventional bus traffic network $\mathrm{A}$ and urban rail traffic network B, respectively, the weight of each edge is defined as the number of public stops between two lines.

(2) If there is an opportunity to transfer between conventional bus traffic lines and urban rail traffic lines, we link these two different types of nodes and constitute the coupling edges of bilayer coupled public traffic network. The coupling edges reflect the transfer relationship between urban rail traffic lines and conventional bus traffic lines. The conventional bus traffic network, urban rail traffic network, and its coupling edges form a bilayer coupled public traffic network.

In this model, the conventional bus traffic network has larger scale but the transmission performance of the network is poor, and the urban rail traffic network is with smaller network size but the network transmission performance is stronger. These two networks coupled together through the transfer relationship and collaboration complete the transport mission of the whole urban public traffic network, and the mixed traffic patterns can make the passengers travel more quickly and conveniently.

Without loss of generality, taking two urban rail lines (subway line 1, subway line 2) and eight conventional bus lines (bus numbers 4, 6, 12, 36, 102, 235, 511, and 707) at Xi'an as the network nodes, we established a new bilayer coupled public traffic network model as shown in Figure 1.

\section{Synchronization Analysis of Bilayer Coupled Public Traffic Network}

We can use the synchronization theory of two different complex networks with time-varying delay coupling to analyze the balance problem of bilayer coupled public traffic network. $\mathrm{Wu}$ et al. [21] reached the conclusion that the passenger flow of urban public traffic fulfills the nonlinear behavior. Through the analysis of the global public traffic network, we know that urban public traffic network has the characteristics of BA scale-free networks. Suppose that the passenger flow of eight bus lines and two rail traffic lines both fulfill the Lorenz 


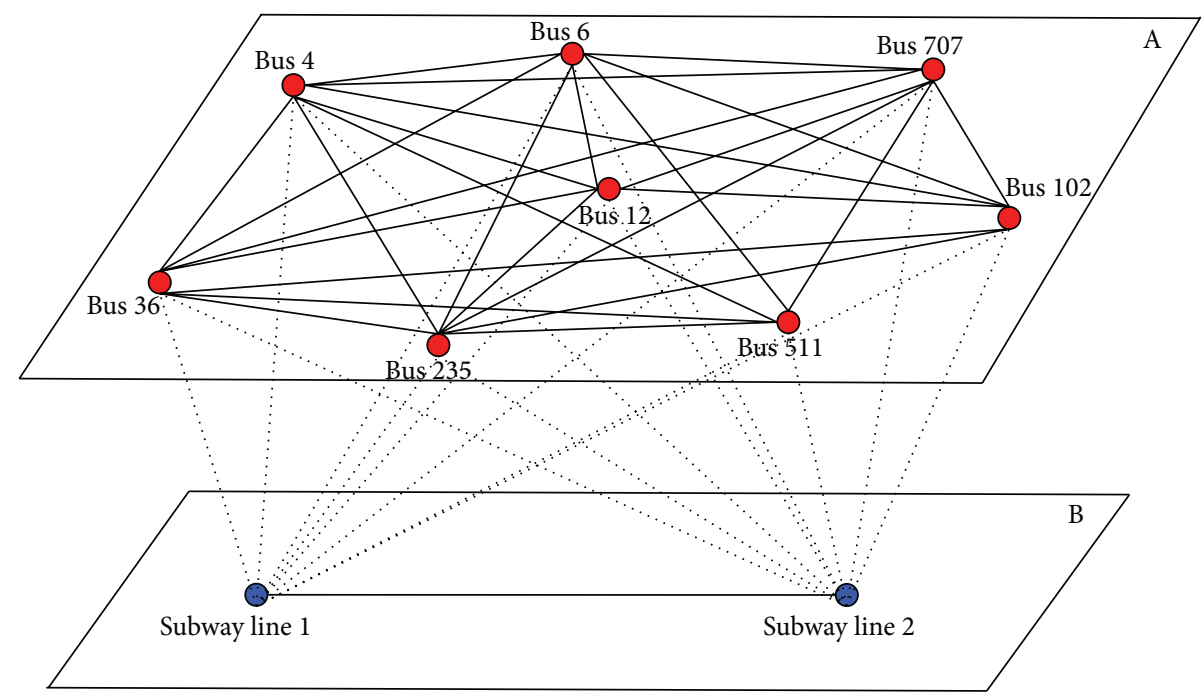

FIGURE 1: The topology map of bilayer public traffic network model.

chaotic system; that is, the nodes dynamical equations can be described as follows:

$$
\left[\begin{array}{l}
\dot{x}_{i 1} \\
\dot{x}_{i 2} \\
\dot{x}_{i 3}
\end{array}\right]=\left[\begin{array}{ccc}
-10 & 10 & 0 \\
28 & -1 & 0 \\
0 & 0 & -\frac{8}{3}
\end{array}\right]\left[\begin{array}{l}
x_{i 1} \\
x_{i 2} \\
x_{i 3}
\end{array}\right]+\left[\begin{array}{c}
0 \\
-x_{i 1} x_{i 3} \\
x_{i 1} x_{i 2}
\end{array}\right],
$$

$$
\left[\begin{array}{l}
\dot{y}_{i 1} \\
\dot{y}_{i 2} \\
\dot{y}_{i 3}
\end{array}\right]=\left[\begin{array}{ccc}
-10 & 10 & 0 \\
28 & -1 & 0 \\
0 & 0 & -\frac{8}{3}
\end{array}\right]\left[\begin{array}{l}
y_{i 1} \\
y_{i 2} \\
y_{i 3}
\end{array}\right]+\left[\begin{array}{c}
0 \\
-y_{i 1} y_{i 3} \\
y_{i 1} y_{i 2}
\end{array}\right] .
$$

For the bilayer coupled public traffic network described in Figure 1, we have the following coupling matrix:

$$
\begin{aligned}
& a_{12}=2, \quad a_{13}=2, \quad a_{14}=2, \quad a_{15}=1, \quad a_{16}=1, \quad a_{17}=7, \quad a_{18}=4, \\
& a_{23}=3, \quad a_{24}=3, \quad a_{25}=1, \quad a_{26}=1, \quad a_{27}=1, \quad a_{28}=3, a_{34}=7, \\
& a_{35}=7, \quad a_{36}=6, \quad a_{37}=0, \quad a_{38}=1, \quad a_{45}=1, \quad a_{46}=2, \quad a_{47}=4, \\
& a_{48}=1, \quad a_{56}=5, \quad a_{57}=0, \quad a_{58}=1, \quad a_{67}=2, \quad a_{68}=1, \quad a_{78}=2, \\
& a_{j i}=a_{i j} \quad(i \neq j, i, j=1,2, \ldots, 8), \quad a_{i i}=-\sum_{i=1, i \neq j}^{8} a_{i j} \quad(i, j=1,2, \ldots, 8), \\
& b_{11}=-1, \quad b_{12}=1, \quad b_{21}=1, \quad b_{22}=-1, \\
& c_{11}=4, \quad c_{12}=3, \quad c_{21}=1, \quad c_{22}=3, \quad c_{31}=6, \quad c_{32}=7, \quad c_{41}=1, \\
& c_{42}=10, \quad c_{51}=2, \quad c_{52}=1, \quad c_{61}=1, \quad c_{62}=3, \quad c_{71}=8, \quad c_{72}=2, \\
& c_{81}=1, \quad c_{82}=1, \quad d_{j i}=c_{i j} \quad(i=1,2, \ldots, 8, j=1,2) .
\end{aligned}
$$

Assume that $\boldsymbol{\Gamma}_{1}=\boldsymbol{\Gamma}_{2}=\operatorname{diag}\{1,1,1\}$ and the controllers are designed as follows:

$$
\begin{aligned}
u_{i}= & \varepsilon_{1} \sum_{j=1}^{2} a_{i j} y_{j}(t-\tau(t))-\varepsilon_{2} \sum_{j=1}^{8} b_{i j} x_{j}(t-\tau(t)) \\
& +\mu \sum_{j=1}^{8} c_{i j} x_{j}(t-\tau(t))-\mu \sum_{j=1}^{2} d_{i j} y_{j}(t-\tau(t))
\end{aligned}
$$

$$
\begin{aligned}
& +\varepsilon_{1} \sum_{j=3}^{8} a_{i j} x_{j}(t-\tau(t))+\varepsilon_{2} \sum_{j=3}^{8} b_{i j} x_{j}(t-\tau(t)) \\
& -\mu \sum_{j=3}^{8}\left(c_{i j}+d_{i j}\right) x_{j}(t-\tau(t))-g_{i} e_{i}(t),
\end{aligned}
$$


According to (1), the dynamical equations of conventional bus traffic network A for each node $i(1 \leq i \leq 8)$ can be described by

$$
\begin{aligned}
& {\left[\begin{array}{l}
\dot{x}_{i 1} \\
\dot{x}_{i 2} \\
\dot{x}_{i 3}
\end{array}\right]=\left[\begin{array}{ccc}
-10 & 10 & 0 \\
28 & -1 & 0 \\
0 & 0 & -\frac{8}{3}
\end{array}\right]\left[\begin{array}{l}
x_{i 1} \\
x_{i 2} \\
x_{i 3}
\end{array}\right]+\left[\begin{array}{c}
0 \\
-x_{i 1} x_{i 3} \\
x_{i 1} x_{i 2}
\end{array}\right]} \\
& +\left[\begin{array}{l}
M_{i 1} \\
M_{i 2} \\
M_{i 3}
\end{array}\right] \\
& M_{1 j}=\varepsilon_{1}\left(a_{11} x_{1 j}(t-\tau(t))+a_{12} x_{2 j}(t-\tau(t))\right. \\
& +a_{13} x_{3 j}(t-\tau(t))+a_{14} x_{4 j}(t-\tau(t)) \\
& +a_{15} x_{5 j}(t-\tau(t))+a_{16} x_{6 j}(t-\tau(t)) \\
& \left.+a_{17} x_{7 j}(t-\tau(t))+a_{18} x_{8 j}(t-\tau(t))\right) \\
& +\mu\left(c_{11} y_{1 j}(t-\tau(t))+c_{12} y_{2 j}(t-\tau(t))\right)
\end{aligned}
$$$$
j=1,2,3
$$

$$
\begin{aligned}
& M_{2 j}=\varepsilon_{1}\left(a_{21} x_{1 j}(t-\tau(t))+a_{22} x_{2 j}(t-\tau(t))\right. \\
& \quad+a_{23} x_{3 j}(t-\tau(t))+a_{24} x_{4 j}(t-\tau(t)) \\
& \quad+a_{25} x_{5 j}(t-\tau(t))+a_{26} x_{6 j}(t-\tau(t)) \\
& \left.\quad+a_{27} x_{7 j}(t-\tau(t))+a_{28} x_{8 j}(t-\tau(t))\right) \\
& \quad+\mu\left(c_{21} y_{1 j}(t-\tau(t))+c_{22} y_{2 j}(t-\tau(t))\right),
\end{aligned}
$$$$
j=1,2,3
$$

$$
\begin{aligned}
& M_{3 j}=\varepsilon_{1}\left(a_{31} x_{1 j}(t-\tau(t))+a_{32} x_{2 j}(t-\tau(t))\right. \\
& \quad+a_{33} x_{3 j}(t-\tau(t))+a_{34} x_{4 j}(t-\tau(t)) \\
& \quad+a_{35} x_{5 j}(t-\tau(t))+a_{36} x_{6 j}(t-\tau(t)) \\
& \left.\quad+a_{37} x_{7 j}(t-\tau(t))+a_{38} x_{8 j}(t-\tau(t))\right) \\
& \quad+\mu\left(c_{31} y_{1 j}(t-\tau(t))+c_{32} y_{2 j}(t-\tau(t))\right),
\end{aligned}
$$$$
j=1,2,3
$$

$$
\begin{aligned}
& M_{4 j}=\varepsilon_{1}\left(a_{41} x_{1 j}(t-\tau(t))+a_{42} x_{2 j}(t-\tau(t))\right. \\
& \quad+a_{43} x_{3 j}(t-\tau(t))+a_{44} x_{4 j}(t-\tau(t)) \\
& \quad+a_{45} x_{5 j}(t-\tau(t))+a_{46} x_{6 j}(t-\tau(t)) \\
& \left.\quad+a_{47} x_{7 j}(t-\tau(t))+a_{48} x_{8 j}(t-\tau(t))\right) \\
& \quad+\mu\left(c_{41} y_{1 j}(t-\tau(t))+c_{42} y_{2 j}(t-\tau(t))\right),
\end{aligned}
$$

$$
\begin{aligned}
& M_{5 j}=\varepsilon_{1}\left(a_{51} x_{1 j}(t-\tau(t))+a_{52} x_{2 j}(t-\tau(t))\right. \\
& \quad+a_{53} x_{3 j}(t-\tau(t))+a_{54} x_{4 j}(t-\tau(t)) \\
& \quad+a_{55} x_{5 j}(t-\tau(t))+a_{56} x_{6 j}(t-\tau(t)) \\
& \left.\quad+a_{57} x_{7 j}(t-\tau(t))+a_{58} x_{8 j}(t-\tau(t))\right) \\
& \quad+\mu\left(c_{51} y_{1 j}(t-\tau(t))+c_{52} y_{2 j}(t-\tau(t))\right), \quad j=1,2,3 \\
& \quad+a_{63} x_{3 j}(t-\tau(t))+a_{64} x_{4 j}(t-\tau(t)) \\
& \quad+a_{65} x_{5 j}(t-\tau(t))+a_{66} x_{6 j}(t-\tau(t)) \\
& \left.\quad+a_{67} x_{7 j}(t-\tau(t))+a_{68} x_{8 j}(t-\tau(t))\right) \\
& \quad+\mu\left(c_{61} y_{1 j}(t-\tau(t))+c_{62} y_{2 j}(t-\tau(t))\right),
\end{aligned}
$$

$$
\begin{aligned}
& M_{7 j}=\varepsilon_{1}\left(a_{71} x_{1 j}(t-\tau(t))+a_{72} x_{2 j}(t-\tau(t))\right. \\
& +a_{73} x_{3 j}(t-\tau(t))+a_{74} x_{4 j}(t-\tau(t)) \\
& +a_{75} x_{5 j}(t-\tau(t))+a_{76} x_{6 j}(t-\tau(t)) \\
& \left.+a_{77} x_{7 j}(t-\tau(t))+a_{78} x_{8 j}(t-\tau(t))\right) \\
& +\mu\left(c_{71} y_{1 j}(t-\tau(t))+c_{72} y_{2 j}(t-\tau(t))\right), \\
& \quad j=1,2,3
\end{aligned}
$$

$$
\begin{aligned}
& M_{8 j}=\varepsilon_{1}\left(a_{81} x_{1 j}(t-\tau(t))+a_{82} x_{2 j}(t-\tau(t))\right. \\
& +a_{83} x_{3 j}(t-\tau(t))+a_{84} x_{4 j}(t-\tau(t)) \\
& +a_{85} x_{5 j}(t-\tau(t))+a_{86} x_{6 j}(t-\tau(t)) \\
& \left.+a_{87} x_{7 j}(t-\tau(t))+a_{88} x_{8 j}(t-\tau(t))\right) \\
& +\mu\left(c_{81} y_{1 j}(t-\tau(t))+c_{82} y_{2 j}(t-\tau(t))\right), \\
& \quad j=1,2,3 .
\end{aligned}
$$

And based on (2) the dynamical equations of urban rail traffic network B for each node $i(1 \leq i \leq 2)$ can be written as

$$
\left[\begin{array}{l}
\dot{y}_{i 1} \\
\dot{y}_{i 2} \\
\dot{y}_{i 3}
\end{array}\right]=\left[\begin{array}{ccc}
-10 & 10 & 0 \\
28 & -1 & 0 \\
0 & 0 & -\frac{8}{3}
\end{array}\right]\left[\begin{array}{l}
y_{i 1} \\
y_{i 2} \\
y_{i 3}
\end{array}\right]+\left[\begin{array}{c}
0 \\
-y_{i 1} y_{i 3} \\
y_{i 1} y_{i 2}
\end{array}\right]
$$

$$
+\left[\begin{array}{l}
N_{i 1} \\
N_{i 2} \\
N_{i 3}
\end{array}\right]+u_{i},
$$




$$
\begin{aligned}
& N_{1 j}=\mu\left(d_{11} x_{1 j}(t-\tau(t))+d_{12} x_{2 j}(t-\tau(t))\right. \\
& \quad+d_{13} x_{3 j}(t-\tau(t))+d_{14} x_{4 j}(t-\tau(t)) \\
& +d_{15} x_{5 j}(t-\tau(t))+d_{16} x_{6 j}(t-\tau(t)) \\
& \left.\quad+d_{17} x_{7 j}(t-\tau(t))+d_{18} x_{8 j}(t-\tau(t))\right) \\
& \quad+\varepsilon_{2}\left(b_{11} x_{1 j}(t-\tau(t))+b_{12} x_{2 j}(t-\tau(t))\right),
\end{aligned}
$$$$
j=1,2,3
$$$$
N_{2 j}=\mu\left(d_{21} x_{1 j}(t-\tau(t))+d_{22} x_{2 j}(t-\tau(t))\right.
$$$$
+d_{23} x_{3 j}(t-\tau(t))+d_{24} x_{4 j}(t-\tau(t))
$$

$$
\begin{aligned}
& +d_{25} x_{5 j}(t-\tau(t))+d_{26} x_{6 j}(t-\tau(t)) \\
& \left.+d_{27} x_{7 j}(t-\tau(t))+d_{28} x_{8 j}(t-\tau(t))\right) \\
& +\varepsilon_{2}\left(b_{21} x_{1 j}(t-\tau(t))+b_{22} x_{2 j}(t-\tau(t))\right),
\end{aligned}
$$$$
j=1,2,3 \text {. }
$$

For any vectors $x_{i}$ and $y_{i}$ of the Lorenz chaotic system, there exists a positive constant $R$ such that $\left\|x_{i m}\right\| \leq$ $R,\left\|y_{i m}\right\| \leq R(m=1,2,3)$, since the Lorenz chaotic system is bounded in a certain region. Therefore, one has

$$
\begin{aligned}
\left\|f\left(y_{i}\right)-f\left(x_{i}\right)\right\| & =\sqrt{\left(-y_{i 1} y_{i 3}-\left(-x_{i 1} x_{i 3}\right)\right)^{2}+\left(y_{i 1} y_{i 2}-x_{i 1} x_{i 2}\right)^{2}} \\
& =\sqrt{\left(-y_{i 3}\left(y_{i 1}-x_{i 1}\right)-x_{i 1}\left(y_{i 3}-x_{i 3}\right)\right)^{2}+\left(y_{i 2}\left(y_{i 1}-x_{i 1}\right)+x_{i 1}\left(y_{i 2}-x_{i 2}\right)\right)^{2}} \leq \sqrt{2} R\left\|y_{i}-x_{i}\right\|
\end{aligned}
$$

that is, Assumption 2 is satisfied. If we select a proper $\tau(t)$ such that Assumption 3 is satisfied, according to Theorem 5, conventional bus traffic network $A$ and urban rail traffic network B achieved synchronization; namely, the whole bilayer coupled public traffic network system is globally asymptotically stable.

\section{Numerical Simulations}

The meaning of urban public traffic network balance is that there is a dynamic balance between running vehicles and passengers; that is, the running time of public traffic vehicles is closest to preset time (traffic delay is shortest), and meanwhile the retention time of the passengers in traffic stations is shortest [25]. The aim of the study is to investigate the impact of public traffic dispatching, delayed departure, the number of public bus stops between bus lines, and the number of transfer stations between two traffic modes, that is, the impact of coupling strengths $\varepsilon_{1}, \varepsilon_{2}, \mu$, time-varying delay $\tau(t)$, the edge weights of conventional bus traffic network A, the edge weights of urban rail traffic network $B$, and the weights of external coupling edges on the balance of whole bilayer coupled public traffic network. During the numerical simulation process, we choose the initial value conditions as follows:

$$
\begin{gathered}
k_{i}=50, \quad(1 \leq i \leq 2), \\
x_{i}(0)=(0.1+0.3 i, 0.2+0.3 i, 0.3+0.3 i)^{T},
\end{gathered}
$$$$
(1 \leq i \leq 8)
$$$$
y_{i}(0)=(2.5+0.3 i, 2.6+0.3 i, 2.7+0.3 i)^{T} \text {, }
$$

$(1 \leq i \leq 2)$

$$
g_{i}(0)=3.3+0.1 i, \quad(1 \leq i \leq 2)
$$

We fixed $\tau(t)=0.05$, and then take $\varepsilon_{1}=\varepsilon_{2}=$ $\mu=0.2$ and $\varepsilon_{1}=\varepsilon_{2}=\mu=0.3$ and draw the synchronization errors for the bilayer coupled public traffic network, as shown in Figures 2 and 3, respectively. From the simulation results, we can come to the conclusion that the bilayer coupled public traffic network achieves balance in 5 time units when $\varepsilon_{1}=\varepsilon_{2}=\mu=0.2$ and achieves balance in 3 time units when $\varepsilon_{1}=\varepsilon_{2}=\mu=0.3$, respectively. Obviously, the greater the value of coupling strengths $\varepsilon_{1}, \varepsilon_{2}, \mu$, the shorter the time required to balance bilayer coupled public traffic network. Accordingly, the coupling strengths have certain influence on the bilayer coupled public traffic network's synchronization; that is, increase of the artificial scheduling (appropriate adjusting of the departing frequency and time, optimization of the transfer facilities, effective traffic dispersion, etc.) can speed up the bilayer coupled public traffic network's synchronization, and the network can reach steady state faster.

Let $\varepsilon_{1}=\varepsilon_{2}=\mu=0.3$ and plot the synchronization errors for the bilayer coupled public traffic network with $\tau(t)=$ 0.03 , as shown in Figure 4. Figure 4 shows that the bilayer coupled public traffic network achieves balance in 2 time units when reducing the delay; compared with Figure 3 the synchronization time decreased 1 time unit. Obviously, the coupling delays have some influences on the bilayer coupled public traffic network's synchronization. This suggests that bus delays or traffic jams caused by the weather or human factors make the passengers stranded time extended, so the time of the network into balance has delayed. 


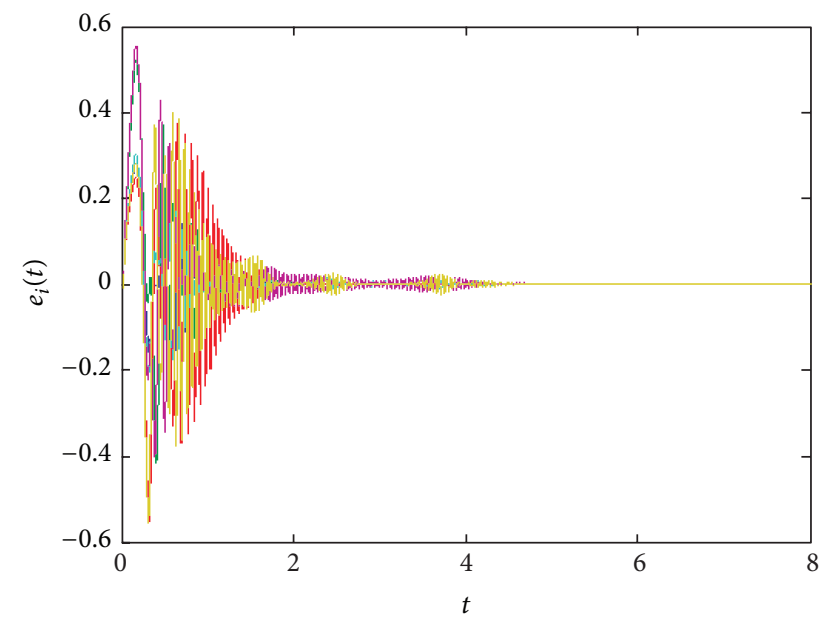

FIGURE 2: Synchronization errors for bilayer coupled public traffic network with $\varepsilon_{1}=\varepsilon_{2}=\mu=0.2$.

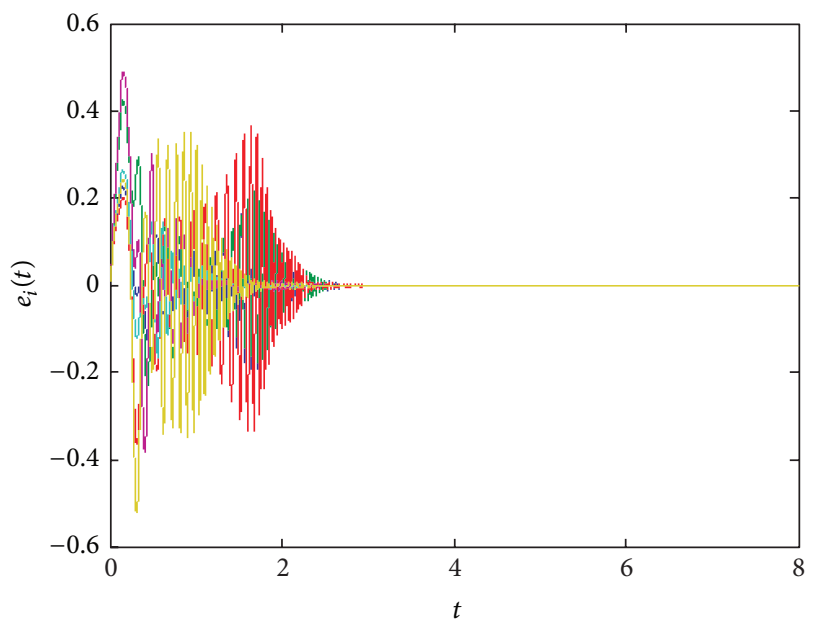

FIGURE 3: Synchronization errors for bilayer coupled public traffic network with $\varepsilon_{1}=\varepsilon_{2}=\mu=0.3$.

Fixing $\tau(t)=0.05, \varepsilon_{1}=\varepsilon_{2}=\mu=0.3$, if the edge weights of conventional bus traffic network $A$ are changed to

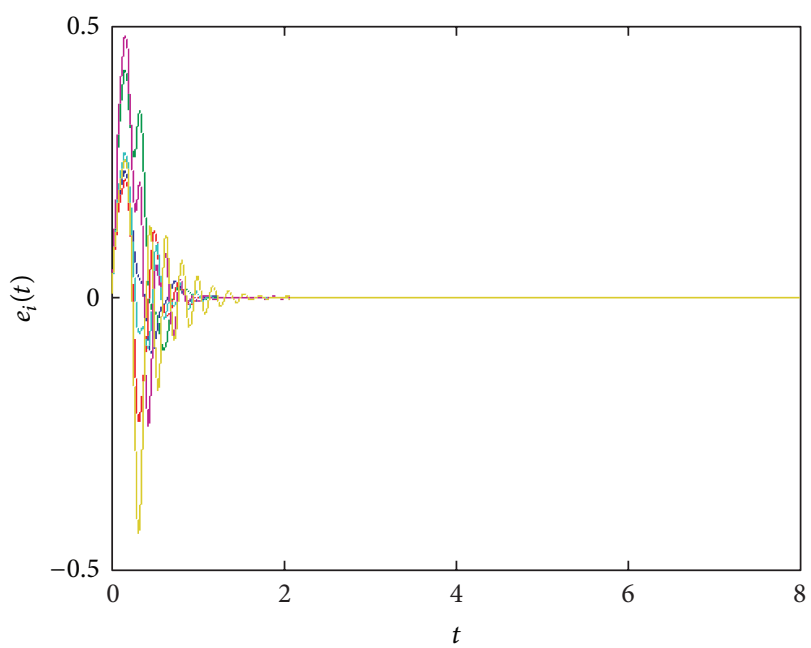

FIGURE 4: Synchronization errors for bilayer coupled public traffic network with $\tau(t)=0.03$.

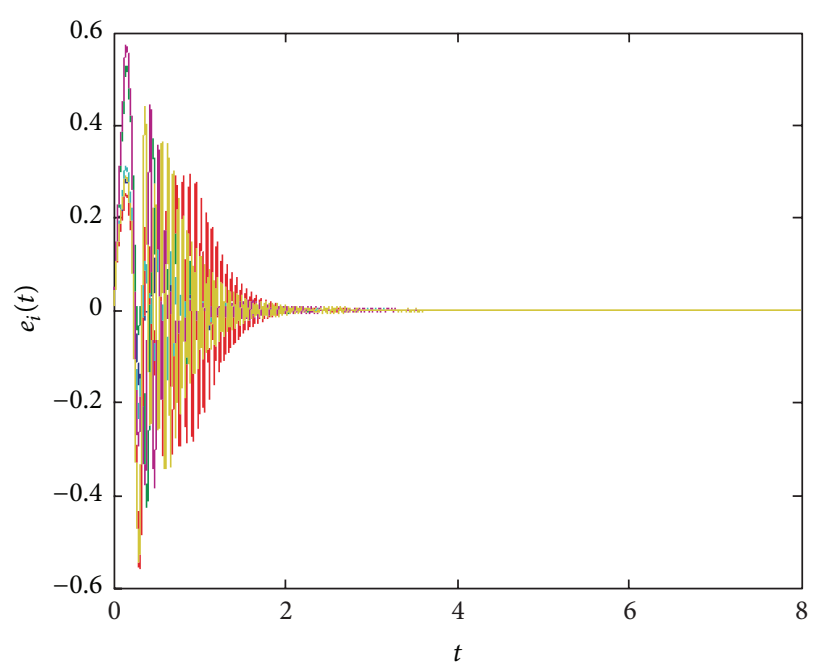

FIGURE 5: Synchronization errors for bilayer coupled public traffic network after decrease of the public bus stops between conventional bus lines.

$$
\begin{aligned}
& a_{12}=0, a_{13}=1, a_{14}=1, a_{15}=0, a_{16}=0, a_{17}=3, a_{18}=2 \text {, } \\
& a_{23}=1, a_{24}=1, a_{25}=0, a_{26}=0, a_{27}=0, a_{28}=1, a_{34}=3 \text {, } \\
& a_{35}=3, a_{36}=3, a_{37}=0, a_{38}=0, a_{45}=0, a_{46}=1, a_{47}=2 \text {, } \\
& a_{48}=0, a_{56}=2, a_{57}=0, a_{58}=0, a_{67}=1, a_{68}=0, a_{78}=1 \text {, } \\
& a_{j i}=a_{i j} \quad(i \neq j, i, j=1,2, \ldots, 8), a_{i i}=-\sum_{i=1, i \neq j}^{8} a_{i j} \quad(i, j=1,2, \ldots, 8),
\end{aligned}
$$

namely, decreased the public bus stops between some conventional bus lines, the synchronization errors are shown in Figure 5. We can found that the bilayer coupled public traffic network achieves balance in 3.6 time units, compared 


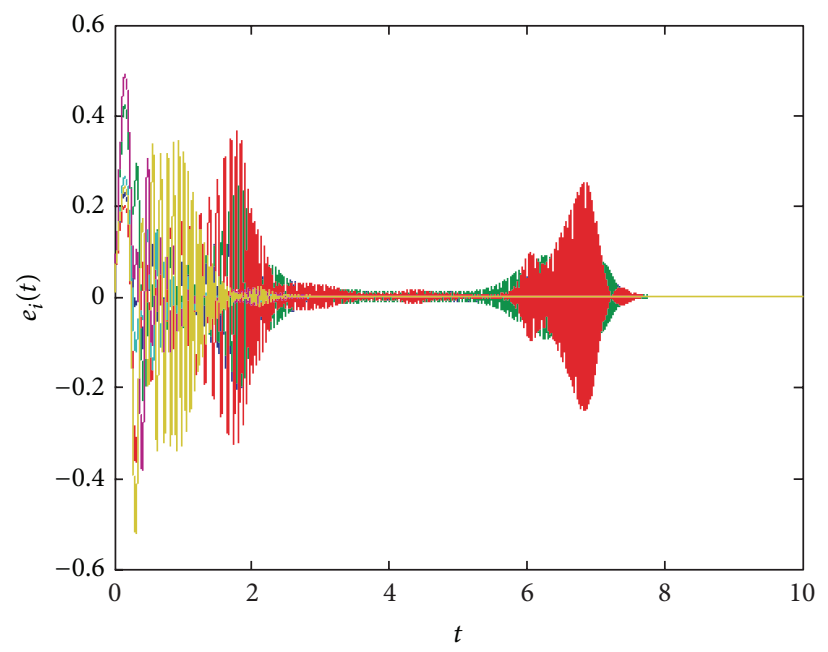

FIGURE 6: Synchronization errors for bilayer coupled public traffic network after reducing the public subway stations between subway lines.

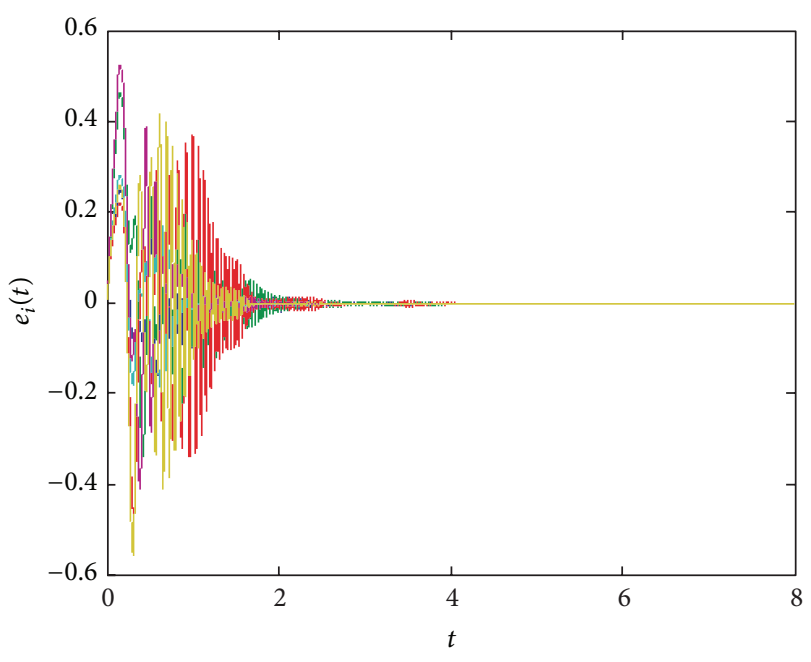

FIGURE 7: Synchronization errors for bilayer coupled public traffic network after decrease of the transfer stations between conventional bus lines and subway lines. with Figure 3 the synchronization time increased 0.6 time units. This implies that the more public bus stops between conventional bus lines the more vehicles for the passengers to transfer, the passengers stranded time have shortened and the faster the whole network reach stable.

Fixing $\tau(t)=0.05, \varepsilon_{1}=\varepsilon_{2}=\mu=0.3$, if the edge weights of urban rail traffic network $\mathrm{B}$ are changed to $b_{11}=0, b_{12}=$ $0, b_{21}=0, b_{22}=0$, namely, remove the public subway stations between subway line 1 and subway line 2 and make no transfer between two subway lines, the synchronization errors are shown in Figure 6. The simulation result shows that the bilayer coupled public traffic network achieves balance in 8 time units; compared with Figure 3 the synchronization time increased 5 time units. This indicates that establishing the public subway stations between two subway lines has very good effect on the whole traffic networks' balance.

Fixing $\tau(t)=0.05, \varepsilon_{1}=\varepsilon_{2}=\mu=0.3$, if the weights of external coupling edges are changed to

$$
\begin{aligned}
& c_{11}=3, c_{12}=2, c_{21}=0, c_{22}=2, c_{31}=5, c_{32}=6, c_{41}=0 \text {, } \\
& c_{42}=9, c_{51}=1, c_{52}=0, c_{61}=0, c_{62}=2, c_{71}=7, c_{72}=1 \text {, } \\
& c_{81}=0, \quad c_{82}=0, d_{j i}=c_{i j} \quad(i=1,2, \ldots, 8, j=1,2),
\end{aligned}
$$

namely, decrease the transfer stations between conventional bus lines and subway lines, the synchronization errors are shown in Figure 7. The result shows that the bilayer coupled public traffic network achieves balance in 4 time units; compared with Figure 3 the synchronization time increased 1 time unit. This shows that the more the transfer stations between conventional bus lines and subway lines, the more the convenience for the passengers to transfer. The passengers stranded time will be shortened, and the bilayer coupled public traffic network can reach balance faster.

\section{Conclusions}

The paper established a bilayer coupled public traffic network model by using the space $R$ modeling method. Based on the synchronization theory of coupling network with timevarying delay, the paper investigated the synchronization of bilayer coupled public traffic network. And the impacts of public traffic dispatching, delayed departure, the number of public bus stops between bus lines, and the number of transfer stations between two traffic modes on the bilayer 
coupled public traffic network balance are analyzed in detail. The results prove that the reasonable artificial scheduling such as appropriate adjusting of the departing frequency and time, optimization of the transfer facilities, and effective traffic dispersion can speed up the bilayer coupled public traffic network's stability. And increase of the public bus stops between conventional bus lines and the public subway stations between subways lines can shorten the passengers stranded time and decreased the whole network synchronization's time. Besides, increase of the transfer stations between conventional bus lines and subway lines can make the bilayer coupled public traffic network reach balance faster. To ease traffic congestion and optimize the traffic network structure, these conclusions can provide decision-making basis for transportation planning, control, and management.

\section{Competing Interests}

The authors have declared that no competing interests exist.

\section{Acknowledgments}

The authors gratefully acknowledge the support from the National Natural Science Foundation of China (no. 61164003, no. 61364001) and Lanzhou Jiaotong University Yong Scientific Research Fund Project (no. 2014024), government of China.

\section{References}

[1] A. Arenas, A. Díaz-Guilera, J. Kurths, Y. Moreno, and C. Zhou, "Synchronization in complex networks," Physics Reports, vol. 469, no. 3, pp. 93-153, 2008.

[2] M. Liu and J. Zhang, "Exponential synchronization of general chaotic delayed neural networks via hybrid feedback," Journal of Zhejiang University-Science A, vol. 9, no. 2, pp. 262-270, 2008.

[3] L. Ling, L. Gang, G. Li et al., "Generalized chaos synchronization of a weighted complex network with different nodes," Chinese Physics B, vol. 19, no. 8, Article ID 080507, 2010.

[4] J. Stout, M. Whiteway, E. Ott, M. Girvan, and T. M. Antonsen, "Local synchronization in complex networks of coupled oscillators," Chaos, vol. 21, no. 2, Article ID 025109, 5 pages, 2011.

[5] L. Yi and W. Xing-Yuan, "Chaotic synchronization in complex networks with delay nodes by non-delay and delay couplings," Acta Physica Sinica, vol. 62, no. 1, Article ID 018901, 2013.

[6] L. Li, Y. Liu, and Q. Yao, "Robust synchronization of chaotic systems using sliding mode and feedback control," Journal of Zhejiang University-Science C, vol. 15, no. 3, pp. 211-222, 2014.

[7] S. Jiang, G. Cai, S. Cai, L. Tian, and X. Lu, "Adaptive cluster general projective synchronization of complex dynamic networks in finite time," Communications in Nonlinear Science and Numerical Simulation, vol. 28, no. 1-3, pp. 194-200, 2015.

[8] Y. Xu, W. Zhou, J. Fang, W. Sun, and L. Pan, "Adaptive synchronization of stochastic time-varying delay dynamical networks with complex-variable systems," Nonlinear Dynamics, vol. 81, no. 4, pp. 1717-1726, 2015.

[9] K. Li, W. Sun, M. Small, and X. Fu, "Practical synchronization on complex dynamical networks via optimal pinning control," Physical Review E, vol. 92, no. 1, Article ID 010903, 2015.
[10] Y. Wang and T. Li, "Synchronization of fractional order complex dynamical networks," Physica A: Statistical Mechanics and Its Applications, vol. 428, pp. 1-12, 2015.

[11] C. Li, W. Sun, and J. Kurths, "Synchronization between two coupled complex networks," Physical Review E, vol. 76, no. 4, Article ID 046204, 2007.

[12] H. Tang, L. Chen, J.-A. Lu, and C. K. Tse, "Adaptive synchronization between two complex networks with nonidentical topological structures," Physica A: Statistical Mechanics and Its Applications, vol. 387, no. 22, pp. 5623-5630, 2008.

[13] J. R. Chen, L. C. Jiao, J. S. Wu, and X. H. Wang, "Adaptive synchronization between two different complex networks with time-varying delay coupling," Chinese Physics Letters, vol. 26, no. 6, Article ID 060505, 2009.

[14] J. Wang, H. Liu, and X. Shi, "Adaptive synchronization between two different complex networks with time-varing delay coupling," Journal of University of Science and Technology Beijing, vol. 327, no. 10, pp. 1372-1378, 2010.

[15] M. Sun, C. Zeng, and L. Tian, "Linear generalized synchronization between two complex networks," Communications in Nonlinear Science and Numerical Simulation, vol. 15, no. 8, pp. 2162-2167, 2010.

[16] Y. Sun, W. Li, and D. Zhao, "Outer synchronization between two complex dynamical networks with discontinuous coupling," Chaos, vol. 22, no. 4, Article ID 043125, 8 pages, 2012.

[17] W. Sun and S. Li, "Generalized outer synchronization between two uncertain dynamical networks," Nonlinear Dynamics, vol. 77, no. 3, pp. 481-489, 2014.

[18] W. Sun, Y. Wu, J. Zhang, and S. Qin, "Inner and outer synchronization between two coupled networks with interactions," Journal of the Franklin Institute, vol. 352, no. 8, pp. 3166-3177, 2015.

[19] B. Wang, W. L. Wang, and X. H. Yang, "An optimal bus transport transfer algorithm based on weighted complex networks," Journal of Wuhan University of Technology, vol. 32, no. 6, pp. 1113-1116, 2008.

[20] W. Hui and H. Wang, "Empirical analysis of complex networks in public traffic networks," Computer Technology and Development, vol. 18, no. 11, pp. 217-222, 2008.

[21] J. J. Wu, Z. Y. Gao, N. H. J. Sun et al., Urban Traffic System Complexity-the Method of Complex Networks and Its Application, Science Press, Beijing, China, 2010.

[22] L. Zhao, M. Deng, J. Q. Wang et al., "Structural property analysis of urban street networks based on complex network theory," Geography and Geo-Information Science, vol. 26, no. 5, pp. 1115, 2010.

[23] J. S. Zhao, Z. R. Di, and D. H. Wang, "Empirical research on public transport network of Beijing," Complex Systems and Complexity Science, vol. 2, no. 2, pp. 45-48, 2005.

[24] M. Chang and S. F. Ma, "Empirical analysis for public transit networks in Chinese cities," Journal of Systems Engineering, vol. 22, pp. 412-418, 2007.

[25] X.-L. An, L. Zhang, and J.-G. Zhang, "Research on urban public traffic network with multi-weights based on single bus transfer junction," Physica A, vol. 436, pp. 748-755, 2015. 


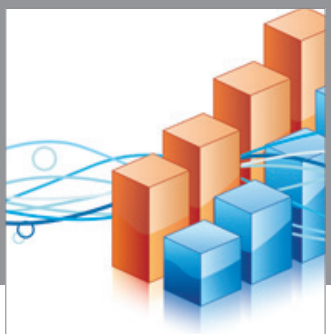

Advances in

Operations Research

vatem alat4

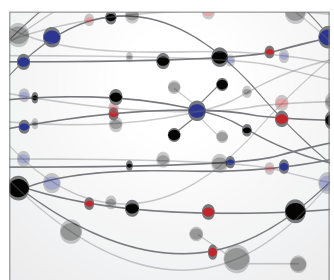

\section{The Scientific} World Journal
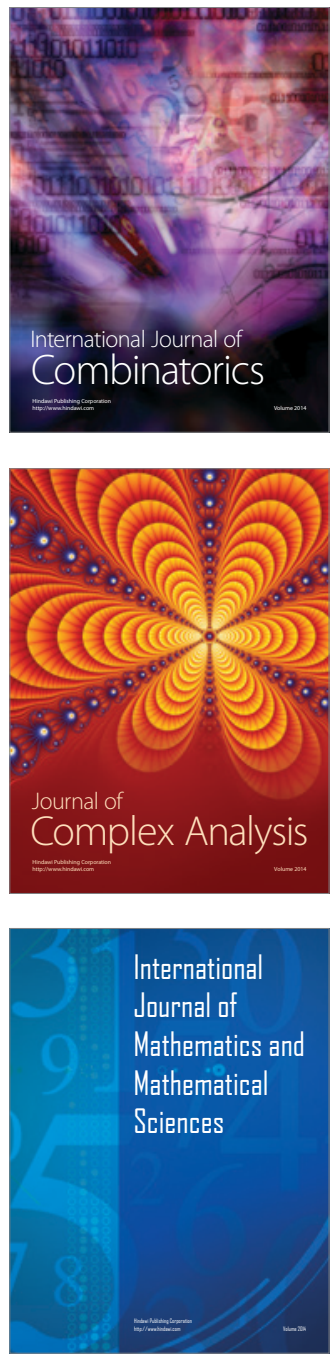
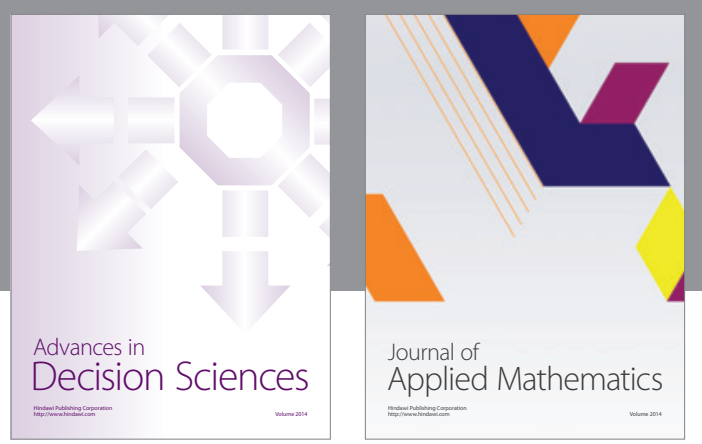

Algebra

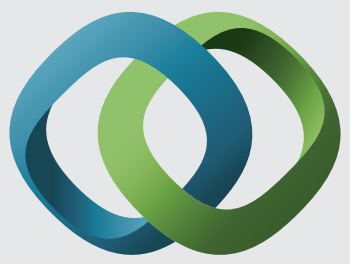

\section{Hindawi}

Submit your manuscripts at

http://www.hindawi.com
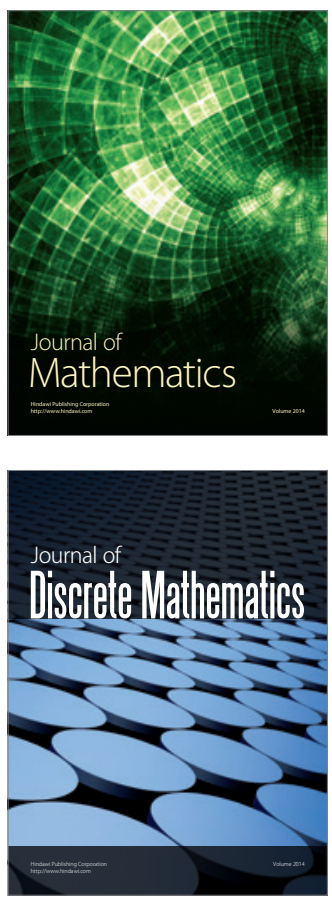

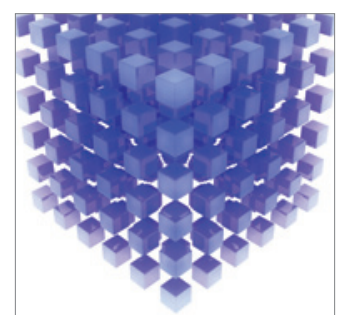

Mathematical Problems in Engineering
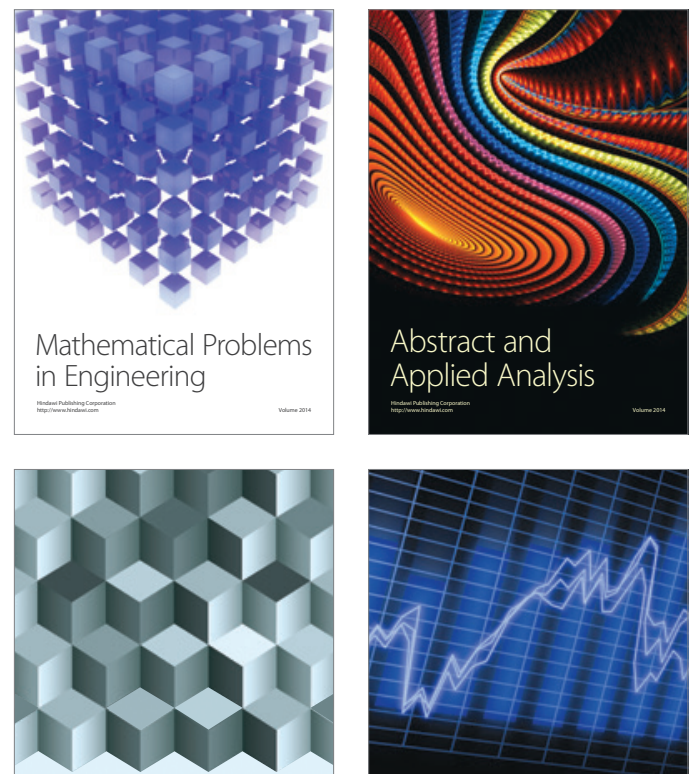

Journal of

Function Spaces

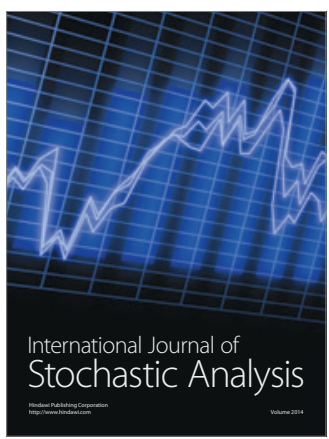

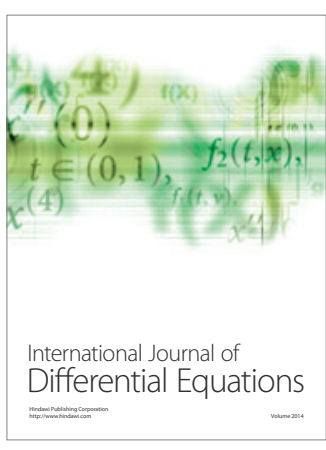
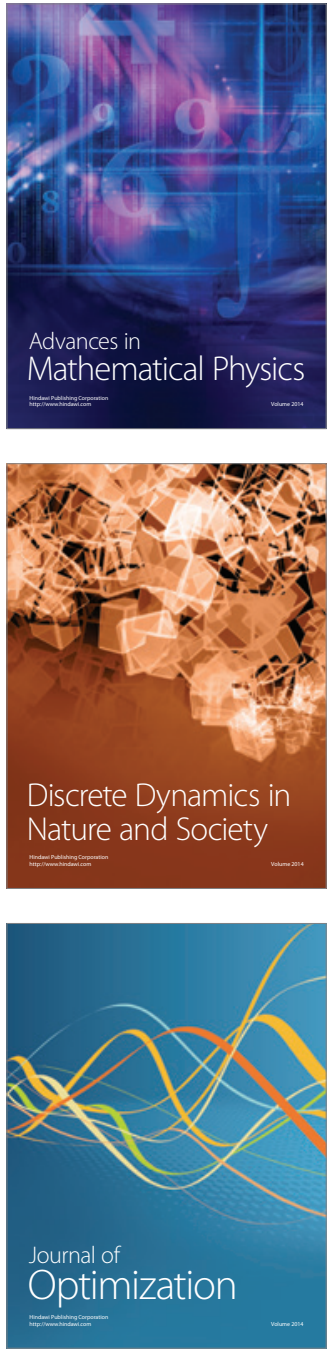